INTERNATIONAL JOURNAL OF

ARTIFICIAL INTELLIGENCE AND EMERGING

TECHNOLOGY



\title{
4D Printing to Develop Egyptian Printing, Packaging and Advertising Sectors
}

\author{
Marwa Mohamed Kamal El Din a, *
}

a Advertising department, Faculty of Applied Arts, Damietta University

\begin{abstract}
$3 D$ printing has been recognized as a disruptive technology for future advanced manufacturing systems. With a great potential, to change everything from our daily lives to the global economy, significant advances in 3D printing technology have been made with respect to materials, printers, and processes. In this context, although similar to $3 D$ printing technology, $4 D$ printing technology adds the fourth dimension of time. $4 D$ printing allows a printed structure to change its form or function with time in response to stimuli such as pressure, temperature, wind, water, and light. Recently, rapid advances in printing processes and materials development for $3 D$ printing have allowed the printing of smart materials or multi materials designed to change function or shape. This paper attempts to go beyond existing capabilities in 4D printing to create precise and universal folding techniques that approach a wider range of applications through a series of radically new physical models. I look into the main factors composing 4D printing technology such as smart materials and designs, then in compare the similarities and differences between $3 D$ printing and $4 D$ printing.

\begin{tabular}{ll}
\hline Keywords & \\
- & $4 D$ Printing \\
- & $3 D$ Printing \\
- & Programming Matter \\
- & Smart Materials
\end{tabular}
\end{abstract}

Introduction

3D printing has captured the imagination of everyone from industry experts to athome hobbyists. Media attention has helped to promote this technology beyond all expectations. The major arguments in favor of 3D printing and Additive Manufacturing are often cited as free complexity, mass-customization and minimizing weight/volume while maximizing strength in components. However, there are significant challenges that need to be addressed in order for 3D printing to have widespread adoption in production and manufacturing, including; printspeed/time, build volume, material quality and new software capabilities. These hurdles have relegated printing to a space of tentative implementation but not yet unanimous 
adoption across industries. A new category of printing has recently been introduced, called 4D printing, which describes the ability for a material system or object to change form and/or function after printing. This technique expands current processes to include the fourth dimension, time, whereby parts can transform themselves in shape or property. 4D printing offers a number of unique advantages over 3D printing that may prove to be the critical capability needed to catalyze widespread implementation. More specifically, 4D printing offers actuation, sensing and programmability embedded directly into a material, without the reliance on external devices and electromechanical systems.

\section{Problem:}

4D Printing as a new technology, the increasingly large market in all over the world and the lack of 4D Printing in Egypt's printing, Packaging and advertising scene are all motivations of this research paper

\section{Methodology:}

The study based on descriptive analyzing researches in order to describe and know how the Egyptian printing technology market will use and able to apply the technology applications in thePrinting, Packaging and Advertising fields.

\section{Goals:}

1. Perform a survey to find out how people feel about the 4D Printing in the Egyptian printingfield and with the normal users.

2. Clarify 4D Printing applications for use, which fulfills the requirements gathered during theresearch

3. Create an effective concept for 4D Printing applications based on the results of the researching 


\section{Programmable Matter}

Programmable matter (PM) is the science, engineering, and design of physical matter that has the ability to change form and/or function (shape, density, moduli, conductivity, color, etc.) in an intentional, programmable fashion. PM may come in at least two forms:

(1) objects made of pre-connected elements that are 4D printed or otherwise assembled as one complete structure for self-transformation.

(2) unconnected voxels that can come together or break apart autonomously to form larger programmable structures. PM encompasses, yet goes beyond, a range of technological capabilities-including 3D printing, micro-robotics, smart materials, nanotechnology, and micro-

electromechanical systems (MEMS), to name a few. And DARPA (The Defense Advanced Research Projects Agency) who has developed PM . ${ }^{8}$

\section{A voxel}

is a volumetric pixel, often used to define the fundamental unit of digital space and Programma- ble Matter. Voxels can be both digital and physical. Digital voxels are computational representations in 3D models. Physical voxels may be comprised of materials as diverse as basic raw materials (e.g., titanium), nanomaterials, integrated circuits, biological materials, and micro-robotics, among others.

\section{4D printing:}

where the fourth dimension entails a change in form or function after 3D printing, is one recent example of PM that allows objects to be 3D printed and then selftransform in shape and material property when exposed to a predetermined stimulus, such as being submerged inwater or exposed to heat, pressure, current, 
ultraviolet light, or other energy source.

PM adds the capability of programming the fundamental materials used in 3D printing and is thus a logical complement and extension of 3D printing

Objects created today, including by 3D printing, are primarily designed to be stable and static- that is, they are unable to change their form or function after fabrication. PM material would change this inert world into a dynamic one, enabling a wide range of capabilities

PM would allow changes in material properties (e.g., flexibility, porosity, conductivity, optical properties, magnetic properties), and it would create objects that could be assembled, disassembled, and then reassembled to form macroscale objects of desired shape and multifunctionality. ${ }^{2}$

This new wrinkle in the maker movement comes courtesy of the Massachusetts Institute of Technology's Self-Assembly Lab, where director Skylar Tibbits and his team are experimenting with so-called "programmable materials." Which based on the PM Technology. The researchers print these substances using a 3-D printer and then watch as the fourth dimension-time-takes over and the materials change shape or automatically reassemble in new patterns. ${ }^{17}$

One vision of the future is PM/4DP with a suite of multiple voxels with different forms and functions that are custom-designed, easily deposited, and then programmed for specific applications. Theoretically, one could have voxels made of metal, plastic, ceramics or any number of other materials. ${ }^{7}$ 


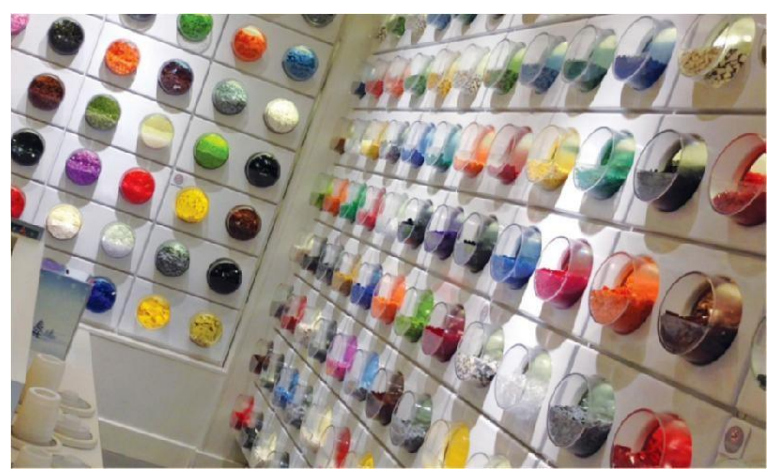

Figure 1: Diversity of bricks in Lego ${ }^{\mathrm{TM}}$ store; Copenhagen, Denmark

\section{Smart Materials:}

Thicker materials change properties slower after being printed but proved to be stronger, whereas thinner materials change quickly but are weaker. When combined, these different material properties react differently to their environments whether they're placed in light, water or some other medium in a way that could mimic the movement of machine-assembled devices driven by actuators, motors and sensors.

The programmed informations embedded inside the form of material's properties or in its shape and the amount of energy used to initiate self-assembly. For example, one of the materials has properties that cause the shape to change and expand if it put in the water. To maintain control over how the material changes, the scientists designed it with a particular geometry that determines the direction it will curl, the number of times it will curl and the angles at which it will curl. ${ }^{9}$ 


\section{Folding structures}

Meanwhile, researchers at Lawrence Livermore National Laboratory have 3D printed shape- shifting structures that can fold and unfold themselves, or expand and contract in size, when prompted by changes in electricity or heat (Fig. 2)

The primary shapes they created were printed with shape-memory polymer inks developed by the team using a direct-ink writing 3D printing process. The ink is made from soybean oil and additional co-polymers, plus carbon nano fibers. ${ }^{18}$

Similarly, a University of Colorado-Boulder team used an Objet Connex multimaterial 3D printerto combine shape-changing materials that respond to heat and printed objects in one shape that changed their shape later, including a selfassembling cube. ${ }^{5}$

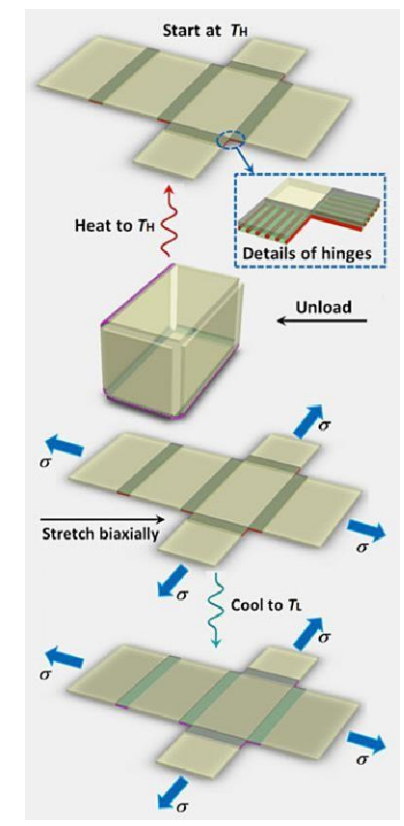




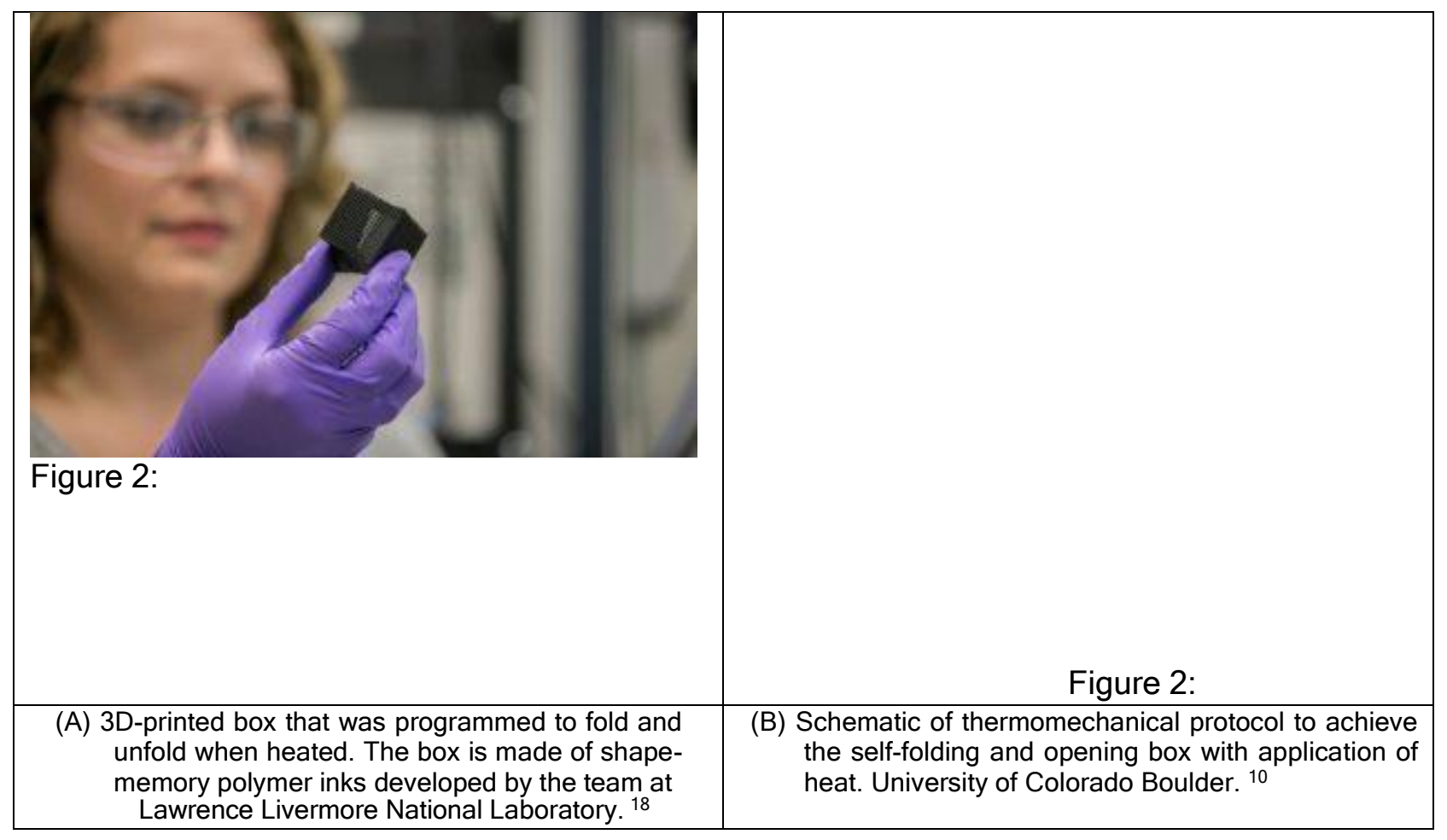

\section{Classification of 3D and 4D Printing Technologies}

The 3D and 4D printing technologies are classified into different printing processes, defined mainly by the types of materials used. The selection of materials has a direct influence on the mechanical or thermal properties, as well as the transformation stimuli of the finished objects.

This section describes the three most common types of $3 D$ and $4 D$ printing and 
reviews the most frequently used materials for these processes. ${ }^{6}$

\subsection{Fused-deposition Modeling (FDM)}

The FDM method operates by extruding thermoplastic materials and placing the semi-molten materials onto a stage to fabricate a 3D structure layer by layer. More specifically, the thermoplastic filament is first led to an extruder which feeds and retracts the filament in precise amounts. The filament is melted by a heater block set to the melting temperature and moved through the extrusion nozzle tip by two rollers. The extruded filament is deposited as the print head traces the design of each defined cross-sectional layer of the desired structure by a digitally positioned mechanism. Then, the stage moves to the $Z$ position in accordance with the setting value of layer thickness. These steps are repeated to complete fabrication of the 3D structure. ${ }^{6}$ 


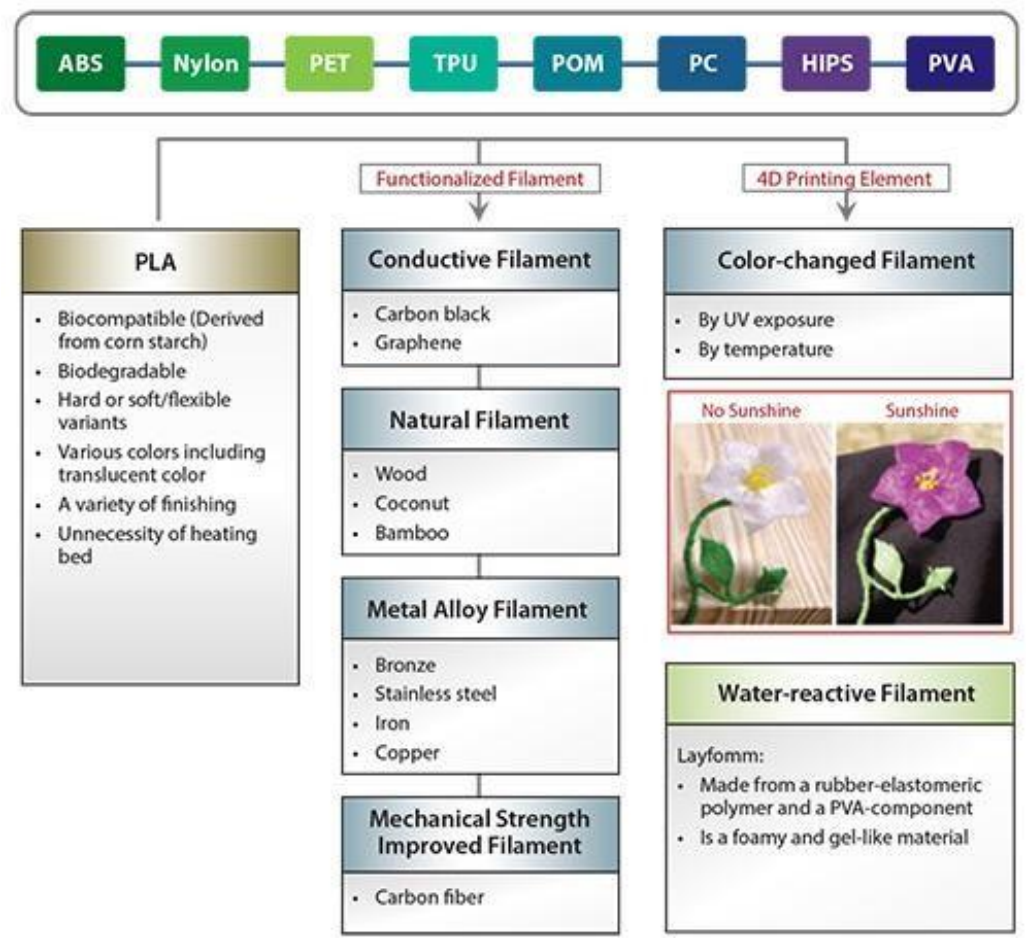

Figure 3: Thermoplastic filaments for Fused-deposition Modeling (FDM). The FDM-printedflower was made with a color changed filament under UV exposure.

\subsection{Powder Bed and Inkjet Head 3D Printing (PBP)}

The PBP process is an adaptation of inkjet printing. In this process, a layer of powder is first deposited and rolled to ensure uniform thickness, then the inkjet print head drops binder in a specified pattern as it moves, to form a single layer of a printed 
object across the bed of powder. The next powder layer is distributed over the deposited liquid binder, and this process is repeated, with each layer adhering to the last. Support structures are not required in PBP due to the ease of removing unbound powder using an air gun, after solidification of the finished object. The use of multiple print heads with colored binder, allows printing in full color. ${ }^{6}$

\subsection{Stereolithography (SLA)}

SLA combines ultraviolet (UV) or visible laser light with curable liquid photopolymer resins. To create each layer, a laser beam illuminates a 2D cross-section of the object in a vat of resin, allowing the resin to solidify. Next, the object is raised by an equal distance of layer thickness to

fill resin under and maintain contact with the bottom of the object. This process is repeated until the entire model is completed, at which point the platform is raised out of the vat and the excess resin is drained. Finally, the SLA object is finished by washing and curing under UV light. SLA produces a smoother surface on the final product compared to other 3D printing methods, as a result of using liquid photopolymers. Although SLA can produce a wide variety of shapes, its drawbacks include a significant amount of resin waste and the need for extensive cleaning after fabrication. Furthermore, resins used in the process are limited to either epoxy or acrylic bases, most of which can shrink upon polymerization. ${ }^{6}$

\section{4D Printing Applications:}

\subsection{Aerial Assemblies}

Aerial Assemblies is part of a series of investigations looking at autonomous assembly in different environments (water, air, space etc). Aerial Assemblies focuses on large-scale modules that can assemble in the airspace high above land, construction sites or complex environments. Each 36" module is made from a helium-filled weather balloon and fiberglass frames. After the helium fades and the 
modules touch-down, the self-assembled lightweight structural lattices will remain. Aerial Assemblies and similarities consider a new idea will open the printing and advertising engineering minds to create a new creative ideas to promote outdoor printing advertising field. ${ }^{11}$


Figure 4: 4D Printing 'Aerial Assemblies'

\subsection{Hyperform}

Hyperform, the winner of the Next Idea Award at Ars Electronica 2013, was a project done in collaboration with Marcelo Coelho and Formlabs. This project addresses the problem of increasingly small 3D print-volumes by identifying a computational and material folding strategy that could allow large scale objects to be compressed into a minimal volume to maximize the printing capability of desktop 3D printers.

While 3D printers are becoming increasingly accessible and capable of rivaling the quality of professional equipment, they are continually limited by smaller and smaller print volumes. This places severe constraints on the type and scale of objects one 
can create. Hyperform offers a new design strategy for relocating density from large objects into small, densely packed, volumes that can be printed then reconfigured into large, functional, objects. A chandelier, eight-times the volume of the 5" cubed 3D print, was produced. A fifty-foot linear chain was also produced from this technique showing the extreme size of objects that are now possible by utilizing computational folding and low-cost 3D Printers.

Hyperform and similarities consider a new idea will open the printing and packaging engineering minds to create a new creative ideas to promote printing packing field, also it will change the structure of folding way and it will supply us with a new ideas to unfold a programmable material within a highly chain. The scientists keep up working on the materials which they lose their ability to change shape after several dozen cycles of folding and unfolding to make them working without losing it. ${ }^{12}$
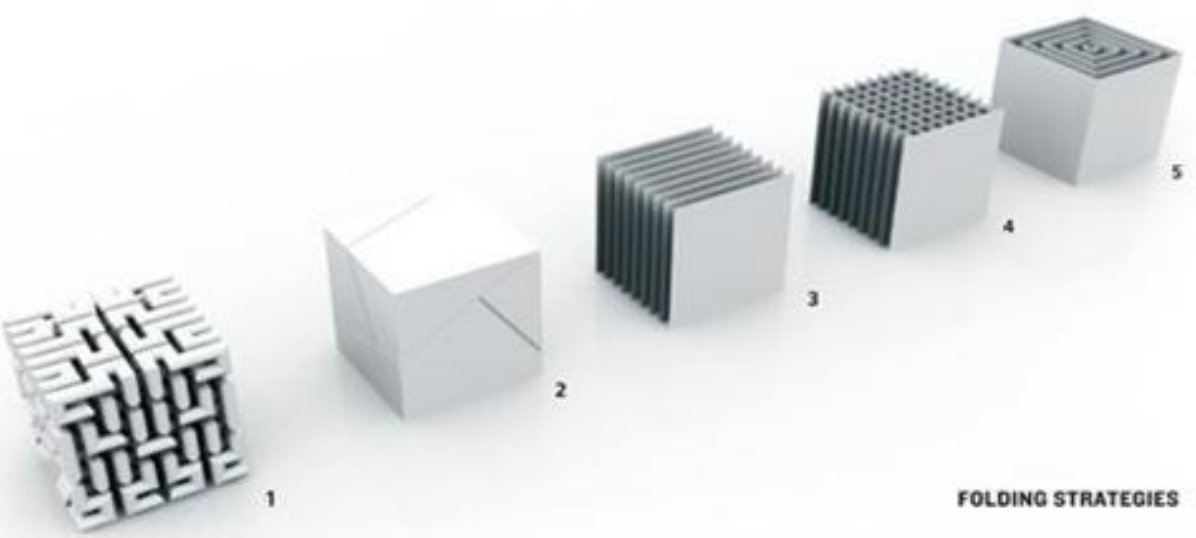

Figure 5: Folding Strategies:

1. HiBert Cube

2. Inspired dissections

3. Cross sectoring 
4. Press $M$

5. Spiral folding

\subsection{Autonomous Mass-Assembly}

This project investigates chiral self-assembly with many parts in order to explore the aggregate behavior of simultaneous assembly and self-selection. Roughly 240 pieces are agitated stochastically to self-assemble closed dodecahedral molecular. Patterns of attraction are designed within each part to specify chemical complementarity and chirality (right and left- handedness). Over time and with the right amount of energy, precise structures emerge complete and self-sorted. This continual process shows the various stages of assembly from independent parts to a mixture of assembled parts, then a bath of fully assembled structures and finally with additional energy input broken again into autonomous pieces. This work points towards a future of both tangible educational tools for non-intuitive scientific phenomena as well as new possibilities for industrial-scaleassembly applications. Autonomous Mass-Assembly and industrial-scaleassembly applications consider a new idea will open the printing and packaging/Advertising engineering minds to create a new creative ideas to promote printing packing/ Advertising field. ${ }^{13}$

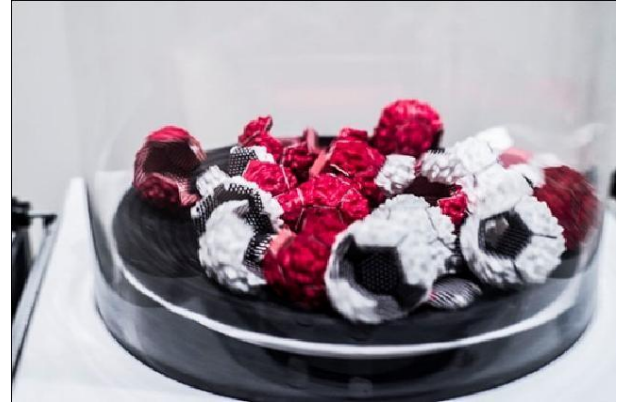

Figure 6: Autonomous

Mass-Assembly 


\subsection{Logic Matter}

Logic Matter as a system of passive mechanical digital logic modules for selfguided-assembly of large-scale structures. As opposed to current systems in selfreconfigurable robotics, Logic Matter introduces scalability, robustness, redundancy and local heuristics to achieve passive assembly. The scientist proposes a mechanical module that implements digital NAND logic as an effective tool for encoding local and global assembly sequences. He/She then show a physical prototype that successfully demonstrates the described mechanics, encoded information and passive self-guided-assembly. Finally, He/She show exciting potentials of Logic Matter as a new system of computing with applications in space/volume filling, surface construction, and 3D circuit assembly.

Logic Matter and surface construction applications consider a new idea will open the printingand packaging/Advertising engineering minds to create a new creative ideas to promote printing packing/ Advertising field. ${ }^{14}$
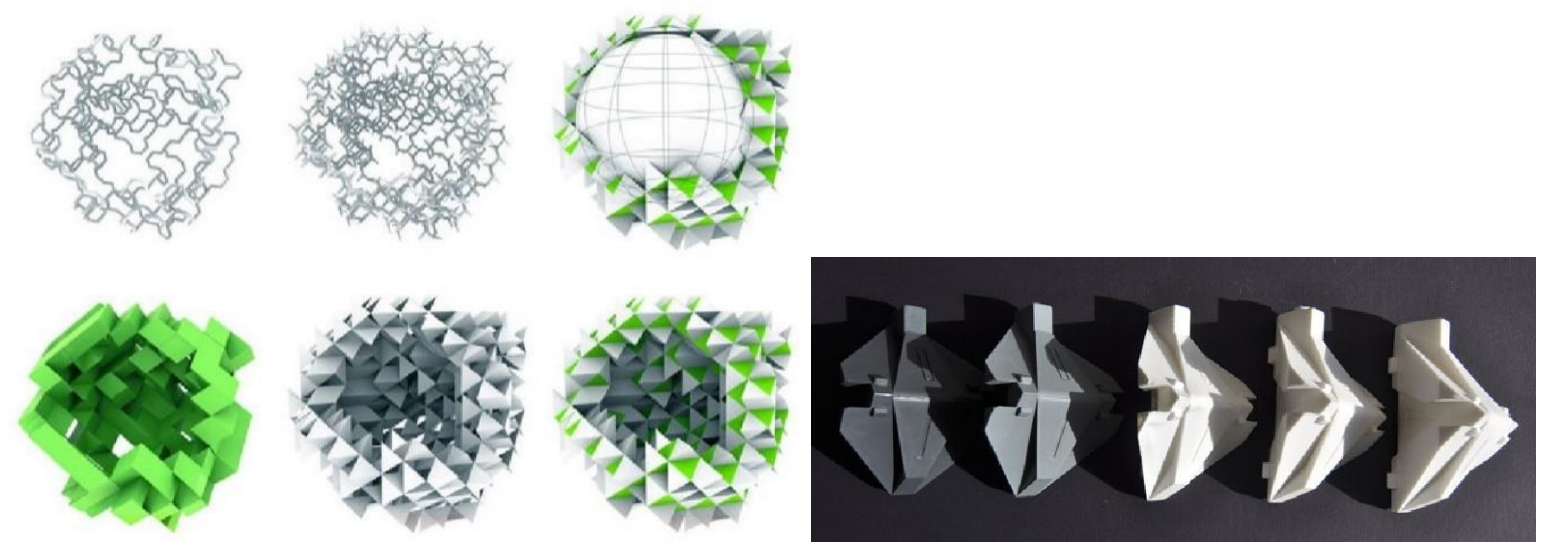

Figure 7: (A) Logic Matter

(B) Physical Mechanism 


\subsection{Single Strand Structures}

The Single-Strand Structures are a series of prototypes looking at building structures with only a single line. Tent poles provide a cheap and quick assembly kit for constructing complex geometry with universal joints. These prototypes follow in a line of research on programmable matter and deconstructing complex geometry into single strand sequences of fold instructions. The Single-Strand Structures similarly ease assembly with a single instruction sequence and allow for fast shipment and deployment of large structures. The assembly kit offers a universal joint for complex vertices of any degree, while also creating a universal hinge, perfect for rigid origami structures. The Hypar surface was constructed from a single $75^{\prime}$ chain of tent pole segments with a tension cord threaded throughout the entire length.

Single-Strand Structures and similarities consider a new idea will open the printing and advertising engineering minds to create a new creative ideas to promote outdoor printing/screenadvertisements structures. ${ }^{15}$ 


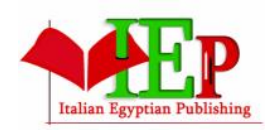

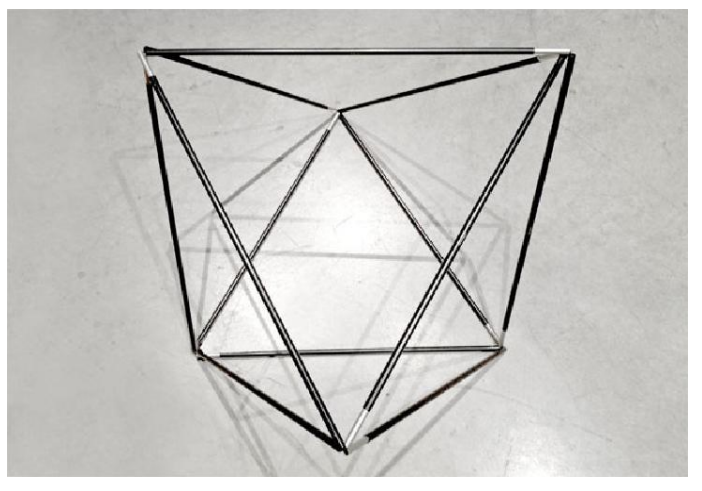
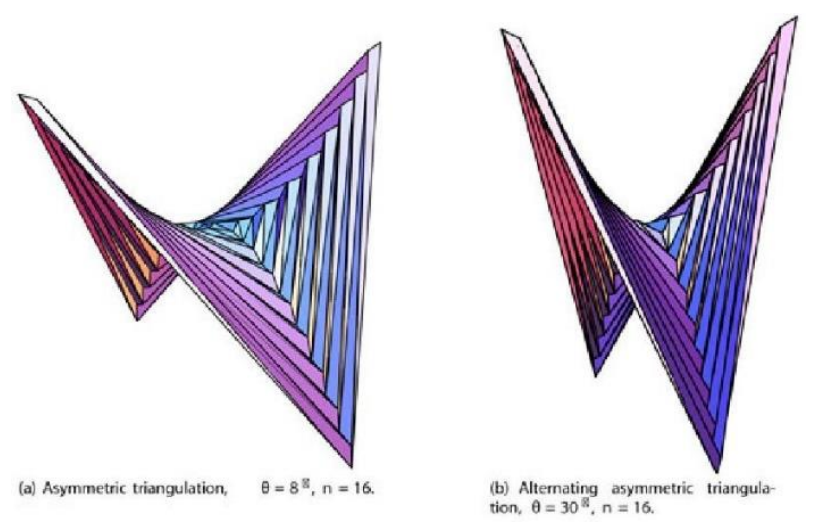

Figure 8: Building structures with single line ' The Hypar surface able to be added as a new structure ways to build indoor/outdoor printed/screen advertising'

\subsection{Some other Examples of 4D Printing}

\subsubsection{Example 1}




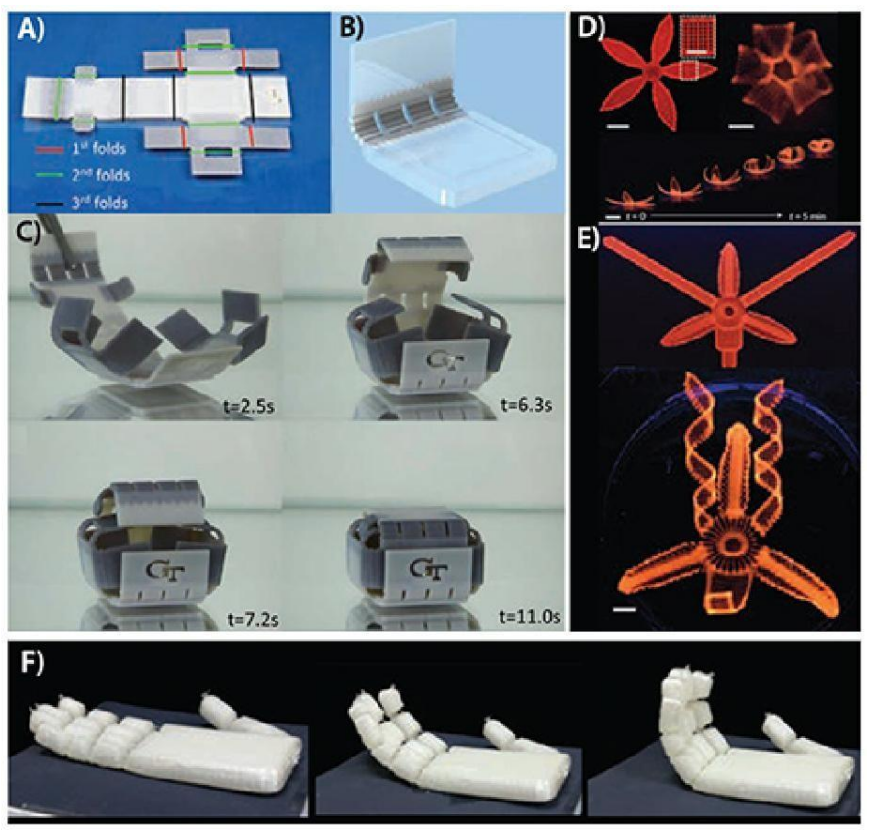

Figure 9. A-B) The design of the folding box with different materials assigned at different hinges. C) Upon heating, the programmed 3D printed sheet folds into a box with a self-locking mechanism. D-E) The resulting swollen flower structures were generated by biomimetic 4D printing with composite hydrogel and cellulose fibrils. F) The temperature-responsive artificial hand

\subsubsection{Example 2}

One recent approach to PM is 4D printing. An example of 4D printed objects that were pre- programmed to respond to a stimulus-water- and change into other shapes is shown in Figure 1. The top figure demonstrates a 1D object morphed into a 2D object- when inserted into water, the snake-like object forms the 
typographic letters "MIT." The bottom two figures demonstrate how one can also create self-folding cubes from both flat and wireframe structures.

\section{4D Printing questionnaire}

Your personal data:

Name:

Your field:

Age:

The researcher has to made a questionnaire to let the users/specializers evaluate the new 4DPrinting technology (samples were taken over 40 person between users and specializers)

4D Printing (4DP) where the fourth dimension entails a change in form or function after 3D printing, is one recent example of PM 'Programmable Matter' that allows objects to be 3D printed and then self-transform in shape and material property when exposed to apredetermined stimulus, such as being submerged in water or exposed to heat, pressure, current, ultraviolet light, or other energy source.

Example: 


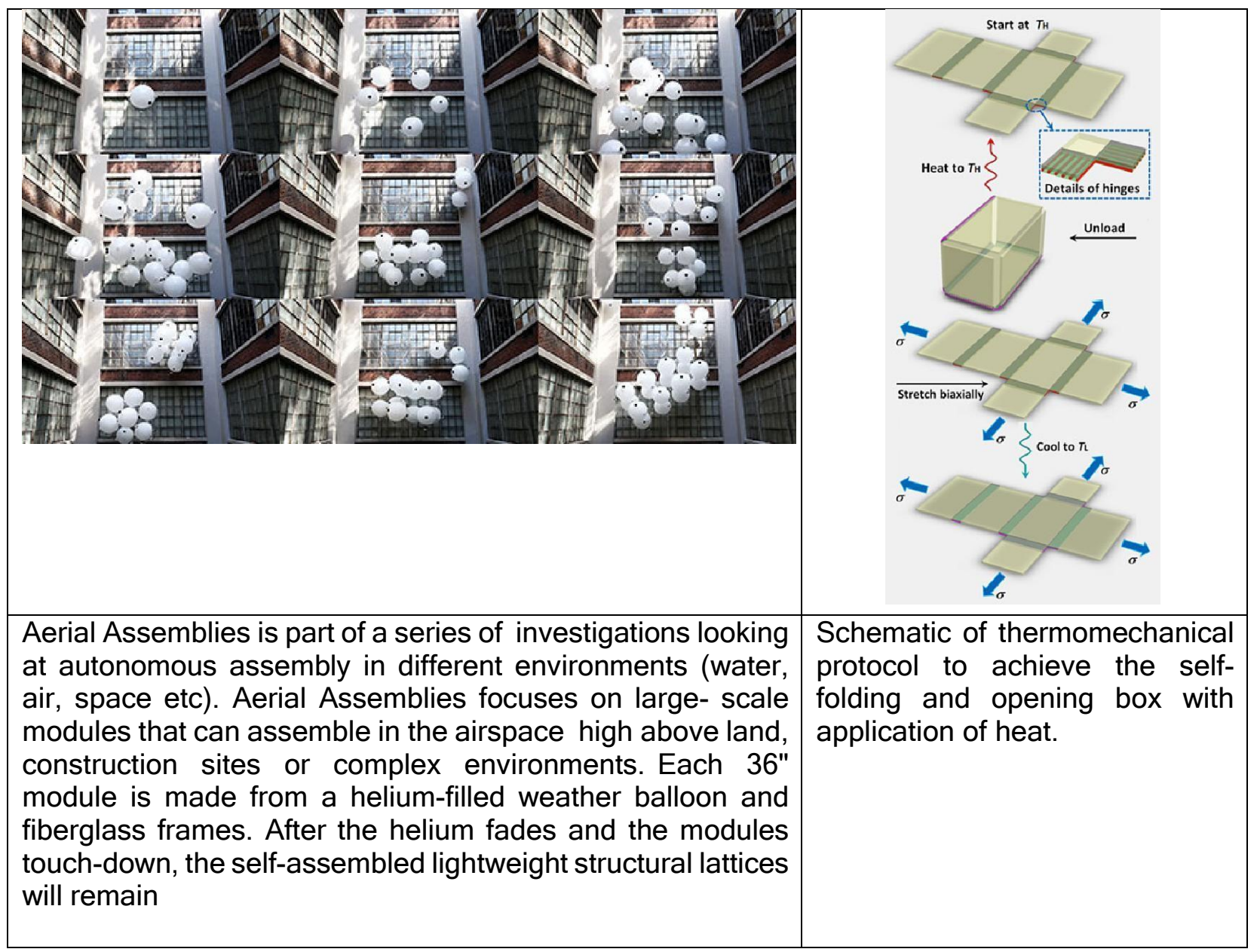

14.1. Users Questionnaire 
packed product opens itself if it put in Ultra Violet for Example?

Do you prefer the

$$
\square \text { Yes } \square \text { No }
$$

Do you prefer the interactivity between you and the programmed products, while you buy it, itcan opened or whatever the programming method which make it open after buying it?

Yes $\square$ No

Do you prefer adding interactivity between you and the advertisement and do you find it a newway of advertising makes people interested in the traditional industries?
$\square$ Yes $\square$ No

\subsection{Specializers Questionnaire}

Do you find the integration between the 4D Printing and the printed media , packaging andadvertising markets a real scientific revolution to promote and marketing the products?
$\square$ Yes $\square$ No

Do you find that adding 4D Printing is an additional value to promote printing, advertising andpackaging fields? 
** Is your firm a sole trader, partnership, private limited company, public limited company, orother (please specify)?

If the company is a member of a group of companies related to media production (printing, packaging, advertising ....Etc.), Has your company ever carried out 4D OR 3D printing and presenting new ideas using those technology possibilities to promote your work, do you intendto add it to your firm in the future??

$\square$ Yes $\square$ No

If yes, please provide details below:

Do you intend to utilize the services you have using one of the new technologies like 4DPrinting?
$\square$ Yes $\square$ No

Do you find using of 4D Printing will make the prints, packed products and advertisementsattractive to users / audience.. do you find it an industry revolution? 
Do you find that programmable materials will help the printing, packaging and advertisingindustry in Egypt to grow up more than what we have now, do you find it useful for the traditionalindustries adv. And packing?
Yes
$\square$ No

Do you find adding self-assembly via 4D Printing to the print will open new markets to yourcompany?
Yes
No

** Please use the following space, if you have any comments on adding 4D Printing as a newtechnology:

\section{Results:}

\section{1. paper results:}

1. 4D Printing, the technology of the future, will change the physical methods of opening and closing packaging products. There are a variety of mechanisms it can offer to develop the packaging world, especially in Egypt

2. 4D Printing as a new technology will change people reacting way with any advertisement.

3. 4D Printing will drive printing, advertising and packaging fields to open new markets to a new packaging/advertisements able to be transformed.

4. The ability to create shape-memory effects like folding, curling, stretching or twistingbased on the orientation and location of particular fibers within composite materials- 
opens up huge possibilities for product design. It may also be possible this shape-altering technology can be adapted to metals and other materials.

5. 4D printing will provide new ways to create highly functional, complex surfaces that could revolutionize Printing engineering.

6. Digital files would be sent anywhere in the world with the right collection of voxels to enable matter formation on demand (Printing matter on demand). With 4D Printing, Extremely simple structures can be printed and then activated by external stimulus to change into complex func- tional structures and systems

7. 4DP Applications shows us several ways of what 4D Printing can do in printing, packaging and advertising fields and how it can turns them totally to be beyond what we can imagine 8. Aerial Assemblies application can be a new way of programmable advertising, and 4D Printing able to develop it more in the near future

9. Logic Matter and Autonomous Mass-Assembly applications are new examples of 4D Printing able to be implement in advertising/packaging fields

10. Hyperform case study is a new successful 4D Printing way to develop packaging world 11. 4D Printing typographic letters morph will lead both indoor/outdoor Advertising to be more easily, functionally and attractive

\subsection{Questionnaire results:}

1. No one of normal users knows any information about 4D Printing or what it can offer to $\mathrm{him} / \mathrm{her}$, but the reaction comes different after they understand it, and they all wants it to start working in both Egyptian packaging and advertising fields.

2. Only $3 \%$ of specialized in Egyptian printing, packaging and advertising field who know about 4D Printing

3. About $90 \%$ of the users prefers to have their programmed products opened or reclose itself and they find it an imaginable and incredible way of dealing with packed products.

4. 4D Printing will open minds with new creative and imaginable ideas in advertising field 
5. Specializers who know 4D Printing find it an additional value and it will promote printing, advertising and packaging fields

6. $72 \%$ of users prefer adding interactivity between them and the advertisement using $4 \mathrm{D}$ Printing, and they find the new ways of advertising makes them interested in the traditional industries and their products and they find 4DP will help revive it again

7. Specializers find $4 D$ Printing a real scientific revolution will promote and marketing the products and will open a new ways of packing traditional products 'or products in general' to send it over the world

8. $78 \%$ of Specializers find adding self-assembly applications of 4D Printing to prints will open new markets to their companies

8. Programmable material will help to develop advertising and packaging fields, especially forthe traditional industries which need a expresses advertisements and creative ideas

\section{Discussion and Finally Conclusion}

Programmable matter and 4D printing certainly have the potential to appear "magical," but they are grounded in real engineering and science research that is only now emerging due to recent technological advances. Several near-term applications of Programmable Matter are on the

horizon. Through programmable matter and 4D printing, there exists the potential to create a new class of disruptive technologies comparable to-and far beyond-3D printing. It does not seem fanciful to imagine a world in which a new form of matter formation could enable form and function modification at the flip of a switch-a world in which one could make intelligent Legolike bricks that can assemble, become multifunctional and morphable into almost any 3D object, and disassemble at will.

Such is the world that is on the horizon with PM. A wide range of technologies are coming together now-software and design tools, new materials, advanced 


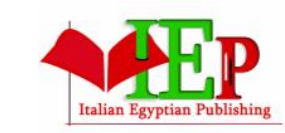

ISSN: $2735-4806$
INTERNATIONAL JOURNAL OF

\section{ARTIFICIAL INTELLIGENCE AND EMERGING}

TECHNOLOGY

VOLUME 1, ISSUE 2, 2018, $23-48$.

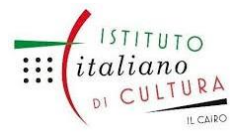

www.egyptfuture.org/ojs/

machines, information, and communication technology (ICT) capabilities, big data, advanced algorithms, and 3D printing- to open new possibilities.

The results indicate that 4D Printing was seen as useful, beneficial, attractive, interesting, and fascinating. People would like to try the applications in both Advertising and Packaging sectors. The expectations placed on 4DP applications are clearly high. 4D Printing considered the coming revolution in printing world which it will help technologies to come and work all together to introduce the best to us. However it will open new opportunities for each sector separately. Step by step it will eliminate some of the traditional production ways, machines and minds. Everything will go to be 4D Printing. I highly recommend using 4D Printing and its future applications in Egyptian printing, advertising and packaging sectors.

\section{References:}

\section{1. papers\& articles}

1. Tibbits, Skylar. 2013. '4D Printing: Multi-Material Shape Change'

2. PM security concerns are analogous to ongoing discussions about the need for enhanced cybersecurity within the Internet of Things (IoT)-e.g., Haynes \& Campbell, 2013

3. 3D printing points are taken from the recent report 3D Printing: More Opportunities Than Dangers, Banning Garrett, Lawrence Livermore National Laboratory.

4. "The Ten Principles of 3D printing" in Fabricated, pp. 20-24.

5. Multimaterial 4D Printing with Tailorable Shape Memory Polymers. Qi Ge, Amir Hosein

Sakhaei ${ }_{2}$ Howon Lee ${ }_{L}$ Conner K. Dunn, Nicholas X. Fang \& Martin L. Dunn. Scientific Reports 18. Ā $\bar{h}$ med Ábdel Azem Mahmoud, Virtual Reality Technology and its Role in Ádvertising Field, International Journal of Multidisciplinary Studies in Art and Technology, Vol. (1), Issue (1), $2018,1-23$.

19. Heba Mohamed Okasha Abou ElKamal, Effect of Implementation 5s Methodology in i'Warehouses at the Egyptian Textiles \& Apparel Factory (Case Study), International Journal of 'Multidisciplinary Studies in Art and Technology, Vol. (1), Issue (1), 2018, 68 - 81. 10. Reem Lotfy Mahmoud Saad, Story Telling and its Relation with Film Script, International

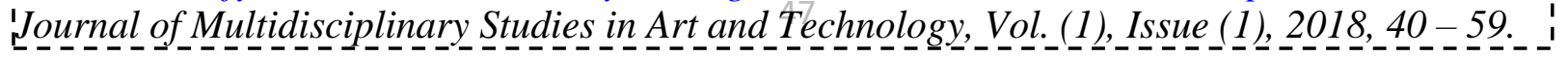


INTERNATIONAL JOURNAL OF

ARTIFICIAL INTELLIGENCE AND EMERGING

TECHNOLOGY

ISSN: $2735-4806$

VOLUME 1, ISSUE 2, 2018, 23 - 48.

www.egyptfuture.org/ojs/

Received: February 15, 2018

Accepted: April 20, 2018 